\title{
The Effect of Field of View on Social Interaction in Mobile Robotic Telepresence Systems
}

\author{
Andrey Kiselev \\ Örebro University, 70182 \\ Örebro, Sweden \\ andrey.kiselev@oru.se
}

\author{
Annica Kristoffersson \\ Örebro University, 70182 \\ Örebro, Sweden \\ annica.kristoffersson@oru.se
}

\author{
Amy Loutfi \\ Örebro University, 70182 \\ Örebro, Sweden \\ amy.loutfi@oru.se
}

\begin{abstract}
One goal of mobile robotic telepresence for social interaction is to design robotic units that are easy to operate for novice users and promote good interaction between people. This paper presents an exploratory study on the effect of camera orientation and field of view on the interaction between a remote and local user. Our findings suggest that limiting the width of the field of view can lead to better interaction quality as it encourages remote users to orient the robot towards local users.
\end{abstract}

\section{Categories and Subject Descriptors}

I.2.9 [Robotics]: Commercial robots and applications; H.5.2 [User interfaces]: Graphical user interfaces (GUI)

\section{Keywords}

Human-Robot Interaction, Mobile Robotic Telepresence, Teleoperation, User Interfaces

\section{INTRODUCTION}

Using mobile robotic telepresence (MRP) systems to interact may lead to undesired situations $[3,4]$ which do not naturally occur in human-human interaction (HHI) and to situations which cannot occur when using static video mediated communication systems. For example, many of the alarm operators learning to maneuver a MRP system while interacting "looked-away" when discussing personal matters with an elderly local user ${ }^{1}$. In HHI, the situation is typically associated with a vis-a-vis configuration $[1,4]$. Authors point out that a possible reason for the unexpected behaviour is that the camera provided the pilot user with a wide field of view. This suggests that the pilot users saw the elderly person without having to orient the robot into the

\footnotetext{
${ }^{1}$ In this paper, we assume that the "local user" is the one located in the same environment as the MRP system, whereas "pilot user" is the one who connects from a remote location.

Permission to make digital or hard copies of part or all of this work for personal or classroom use is granted without fee provided that copies are not made or distributed for profit or commercial advantage, and that copies bear this notice and the full citation on the first page. Copyrights for third-party components of this work must be honored. For all other uses, contact the owner/author(s). Copyright is held by the author/owner(s).

HRI' 14, March 3-6, 2014, Bielefeld, Germany.

ACM 978-1-4503-2658-2/14/03.

http://dx.doi.org/10.1145/2559636.2559799
}

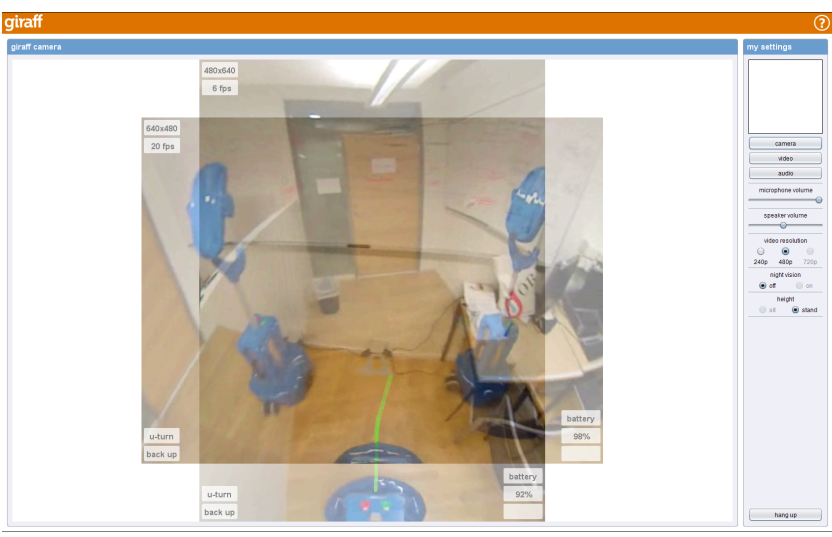

Figure 1: Pilot interfaces with portrait or landscape image orientation.

vis-a-vis position. Whether or not the look-away behaviour is acceptable among real elderly users was investigated in [3]. The elderly, who were guided around in a smart home, were observed to adjust their own position towards a vis-a-vis if the pilot user created the look-away configuration. The importance of being able to see the person they were talking to was emphasized during the retrospective interviews following the interaction.

This study investigates whether providing a wide-angle portrait view instead of a wide-angle landscape view can influence pilots to orient the robot more towards a local user in order to improve quality of interaction. Quality of interaction is measured in terms of orientation of the telepresence robot towards a local user. The results show that pilot users using the MRP system providing a portrait wide-angle view orient the robot more towards the local user than those who use the system with a landscape wide-angle view.

\section{METHOD}

The MRP system used in this experiment was Giraff. Two identical robots with different camera orientations were used. Fig. 1 depicts the landscape and portrait view provided via the Giraff Pilot user interface. The diagonal field of view and the size of the area covered is the same for both robots.

All participants received introductory information about the objectives of the experiment and a 10-minute demonstration session on driving the Giraff providing a cropped squared field of view. The training session served two pur- 


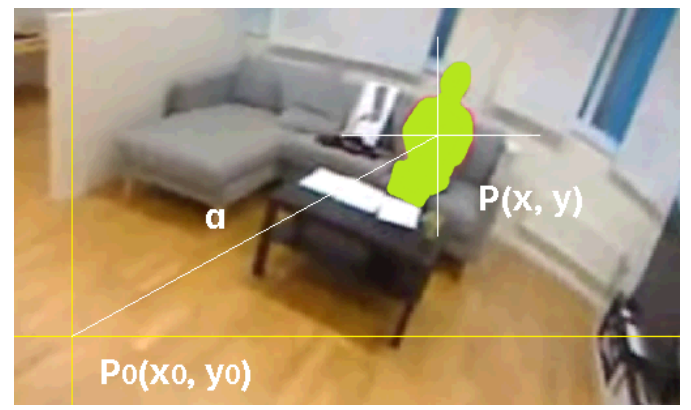

Figure 2: Calculating the angle $\alpha$ using the linear distance between the optical axis of robot's camera $P_{0}$ (center of the image) and the location of the user on the image $P$.

poses: minimize bias and allow the participants to familiarize themselves with the MRP system. Upon completing the training session, two conversational sessions were conducted one after the other. One session using the robot providing a landscape wide-angle view and one session using the robot providing the portrait view. The order of the sessions was mixed across participants.

Conversational sessions were built like parts of interview with general questions about participants' background, without any connection to experiment objectives. During the sessions, the local user (one of experimenters) was always sitting in the same position.

Quality of interaction was measured as the angle between the optical axis of robot's camera and a line from the robot's camera to the local user. Concerning hardware limitations of the robot and based on the fact that the distance from the robot to the local user was always limited in the bounds from two to four meters, the actual angle $\alpha$ was approximated in real time from the image stream received from the robot by measuring the linear distance from the center of the image, $P_{0}$, to the location of the local user, $P$ (see cropped image in Fig. 2). The unit is non-dimensional and ranges from 0 to 1 where 0 corresponds to no angle (absolute vis-a-vis formation) and 1 corresponds to maximum possible angle (local user is still observable, but located in the corner of the image). The $\alpha$ is sampled every $500 \mathrm{~ms}(2 \mathrm{~Hz})$ while the local user is found in the image. The location of the local user was extracted by using thresholding in HSV space based on color samples of the local user's t-shirt.

\section{RESULTS}

Four male students from the School of Science and Technology, Örebro University, Sweden, participated in the experiment. Their age ranged from 19-21 years of age and all had experience of PC/video games in which players move. All conversational sessions lasted from $63 \mathrm{~s}$ to $101 \mathrm{~s}$ ( $\mu=$ $81.7 s, \sigma=13.11 s)$. The median and SD values of $\alpha$ for each participant's conversational sessions are shown in Fig. 3. For each participant, the lowest mean and median values of $\alpha$ were found in the conversational session in portrait mode. Per-participant statistical significance of the results has been verified using one-way ANOVA tests.

The linear distance, $\alpha$ is calculated such that the lowest possible value occurs when the local user is located in the center of the image and the largest values occur when the

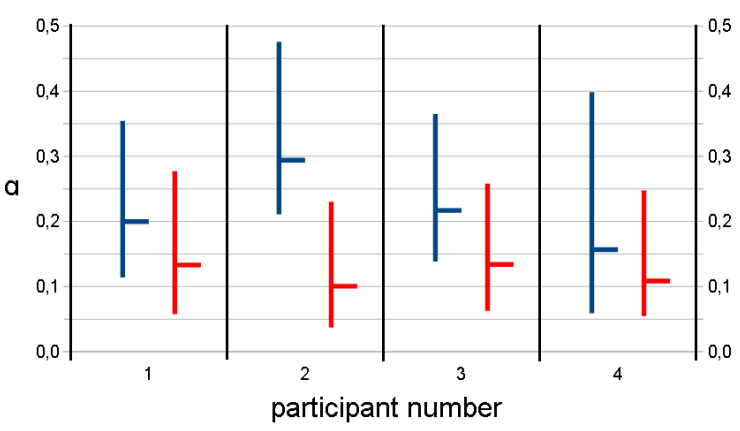

Figure 3: Median and SD values for landscape (blue, left) and portrait (red, right) camera orientation.

local user is located in any of the four extreme corners. Our results show that $\alpha$ is larger in the landscape condition than in the portrait condition. Pilot users provided with a landscape view tend to maintain the conversation with the local user non-centered. Nevertheless, comparable results were found when including only the $\alpha$ located within the area that is common for both views (see Fig. 1).

\section{DISCUSSION AND CONCLUSIONS}

An important result in this study is that an assumption taken by many engineers that a landscape wide-angle view is best, is not supported. By rotating the robot's camera $90^{\circ}$ and limiting the horizontal view angle while increasing the vertical view angle, the $\alpha$ decreased. Drawing on experiences from [3], this increased the quality of interaction from the local user's perspective but additional studies are needed to confirm this hypothesis. While the view provided can also be obtained by cropping the image, it is our belief that the bigger vertical view angle can also improve the driving experience. This is supported by our previous study [2] which shows that the mental workload can be high while driving the robot. By offering the participants a portrait field of view, the need to tilt the screen is eliminated while driving. However, future work includes studying whether there are trade-off's in the pilot's driving capabilities and experienced interaction quality if limiting the width of the view.

\section{REFERENCES}

[1] A. Kendon. Spatial Organization in Social Encounters: The F-formation System. In Conducting Interaction: Patterns of Behavior in Focused Encounters, pages 209-238. Cambridge University Press, 1990.

[2] A. Kiselev and A. Loutfi. Using a Mental Workload Index as a Measure of Usability of a User Interface for Social Robotic Telepresence. In Proceedings of the Ro-Man 2012 Workshop Social Robotic Telepresence, pages 3-6, 2012.

[3] A. Kristoffersson, S. Coradeschi, A. Loutfi, and K. S. Eklundh. Assessment of Interaction Quality in Mobile Robotic Telepresence - An Elderly Perspective. Interaction Studies. in press.

[4] A. Kristoffersson, K. S. Eklundh, and A. Loutfi. Measuring the Quality of Interaction in Mobile Robotic Telepresence A Pilot's Perspective. International Journal of Social Robotics, 5:89-101, 2013. 